\title{
SWOT Analysis in Yoga Research
}

\author{
Kaviraja Udupa ${ }^{1}$, Meena Ramanathan ${ }^{2}$, Ananda B Bhavanani ${ }^{3}$
}

\begin{abstract}
This article is based on the "Open House Discussion on Yoga Research: Strength, weakness, opportunities, and threat (SWOT) analysis" that was organized during the international seminar cum workshop conducted by the Centre for Yoga Therapy, Education and Research (CYTER), an innovative center of Sri Balaji Vidyapeeth (SBV), on "Wholistic Approach of Yoga Therapy in Health and Disease" as part of the International Health Research Convention 2019 organized by SBV in July 2019.

In this article, we have discussed various factors and dimensions of strength, weakness, opportunities, and threats (challenges or risks) in yoga research especially in the Indian context based on the open house discussion. We have also discussed the ways that could be adopted to improve the standard of yoga research to provide more reliable, authentic, and validated data to evidence-based research. Yoga therapy is a newly emerging field; hence, it is important to analyze the inherent strengths that make research in this field useful for a large section of society as well as the weaknesses that may hinder the quality research in yoga therapy. It is also important to find out possible opportunities that can enhance or boost the work done in this field and know the threats that can retard or hamper quality research.
\end{abstract}

Keywords: Challenges, Opportunities, Strength, Threats, Weakness, Yoga therapy.

SBV Journal of Basic, Clinical and Applied Health Science (2021): 10.5005/jp-journals-10082-03101

\section{INTRODUCTION}

Searching intensely to find a solution to a question is research, which may lead you into more such newer queries arising. Strength, weakness, opportunities, and threat (SWOT) analysis is the fourpronged approach to analyze the ongoing productivity of a system. It is commonly used in industry to assess the performance or productivity of a given outlet or unit. Yoga as a lifestyle modification entity has much more to offer to the wholistic well-being of an individual enabling him to achieve and sustain the same eternally. But, in this article, we will be discussing on yoga research that is being practiced in India which is caught between the vastness of yoga philosophy and inability of the modern scientific gadgets to quantify the qualitative "real" improvement produced by yoga due to multiple limitations of scientific studies. By SWOT analysis, we are able to analyze how external opportunities help to combat the internal weaknesses. It helps to minimize the weaknesses so that the identified threats are avoided. So, by this method, we can analyze and achieve the objective-goal. ${ }^{1-6}$

Research in yoga has originated way back in the prehistoric era. The seers even then had started seeking and repeatedly searching answers to the question, "Who am I?" Research in yoga has advanced from what it used to be, but more to be accomplished yet.

It must be kept in mind that research in yoga is not merely a collection of information on yoga or rearranging of known facts and it is also not a mere expansion of previous work done. True research is a search related to a specific question that needs an answer leading to a new question. But sadly, most research studies in yoga in recent times seem either to be for curing diseases and disorders that are widespread or to publicize the techniques of their own systems. The focus needs to be to find newer methods as part of basic research which is lacking in modern times as well as to unravel its secrets on the core concepts of yoga.,

Proper research is an exploration related to a particular problem that needs a solution, further leading on to another question, and so on. But sadly, recent studies in yoga are for treatment and cure
${ }^{1}$ Department of Neurophysiology, NIMHANS, Bengaluru, Karnataka,
India
${ }^{2,3}$ Centre for Yoga Therapy Education and Research (CYTER), Sri Balaji
Vidyapeeth, Puducherry, India

Corresponding Author: Ananda B Bhavanani, Centre for Yoga Therapy Education and Research (CYTER), Sri Balaji Vidyapeeth, Puducherry, India, Phone: +91 9842311433, e-mail: yoga@mgmcri.ac.in

How to cite this article: Udupa K, Ramanathan M, Bhavanani AB. SWOT Analysis in Yoga Research. J Basic Clin Appl Health Sci 2021;4(1):26-30. Source of support: Nil

Conflict of interest: None

of diseases that are widespread or to publicize the techniques of their own systems. The focus needs to be to find newer methods as part of fundamental research which is deficient in current times as well as to unravel its secrets on the core concepts of yoga., Many studies have been done over the past few decades, and an extensive review has reiterated yet again the therapeutic potential on stress-triggered psychosomatic disorders. ${ }^{9}$ Nowadays people are not only simply looking for the treatment of diseases alone but also are concentrating equally on the aspects of prevention and management of these diseases. With the advancements in the growing society, many revolutionary changes have taken place in the modes and methods of treatment. ${ }^{10}$

SWOT analysis is a planning technique that is used to identify strengths, weaknesses, opportunities, and threats, which enables to help a person or an organization associated with business or project planning or any other subject. SWOT analysis is often used to answer questions and generate meaningful information for each category to identify their advantages and is a time-tested tool in strategic analysis (Table 1). Strengths and weaknesses are commonly or more often related to internal issues while opportunities and threats focus on external issues and problems. ${ }^{11}$ 
Table 1: SWOT table suggesting major points of analysis of the ongoing yoga research

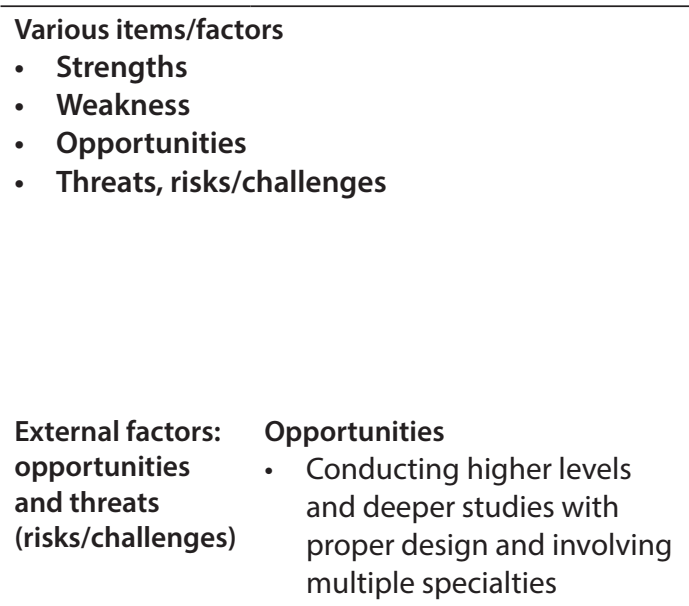

Threats

- Compliance issues to carry out yoga training by participants

- Questionable data collection, analysis, and interpretation

\begin{tabular}{|c|c|}
\hline \multicolumn{2}{|c|}{ Internal factors: strengths and weakness } \\
\hline $\begin{array}{l}\text { Strengths of yoga } \\
\text { - Cultural heritage } \\
\text { - Time tested and effective in } \\
\text { various disorders } \\
\text { - Lifestyle entity with many } \\
\text { facets to training raising } \\
\text { one's consciousness to higher } \\
\text { levels so as to unite with }\end{array}$ & $\begin{array}{l}\text { Weaknesses of yoga research } \\
\text { - Low scientific vigor } \\
\text { - Not well-validated, one cap fitting all } \\
\text { heads approach } \\
\text { - Difficulties in conducting blinded } \\
\text { randomized controlled trials, getting ethics } \\
\text { approval, funding, and publishing in high- } \\
\text { impact journals }\end{array}$ \\
\hline
\end{tabular}
supreme consciousness

\section{Strength and opportunities (SO)} strategies

- Conducting yoga research with good design and in-depth studies not just superficial benefits

\author{
Strength and threats (ST) \\ strategies \\ - Designing study countering all \\ the threats/risks
}

Weakness and opportunities (WO) strategies

- Planning study with proper control group, double-blind, unbiased interpretation and then convincing reviewers of funding agencies and journals with high quality of scientific work

Weaknesses and threats (WT) strategies

- Designing study countering all the weakness, threats/risks

\section{Strengths of Yoga Research}

Yoga being the cultural heritage of India has all along been practiced as a way of life from time immemorial. Focusing on core concepts of yoga, extensive basic research is required to unravel its secrets. Recent literature using modern scientific research has demonstrated its beneficial effects in various medical conditions. However, in a general context, yoga is often misinterpreted and misunderstood by the common man only as a set of asanas (physical postures) and a few techniques of pranayama (breath regulating techniques).

Yoga as a lifestyle comprises of the exhaustive literature enriched with deep philosophy and an entity with eight different limbs to achieve supreme goals known as "Ashtanga Yoga" (moral and ethical values and disciplines; postures to be practiced with breath control and awareness to prepare the body well for a higher order of functioning; meditation and concentrated approaches to unite one's consciousness to higher universal consciousness). ${ }^{12}$

"Proof" that yoga therapy is effective and accepted as a valid treatment enables it to be more widely followed as well as established as an "evidence-based medicine." It paves a path to be tried with respect by both physicians and patients instead of being uncertain. Whereas clinically relevant factors are segregated and focused upon by scientific research and these findings develop an ability to compare yoga knowledge with allopathic medicine and enable clearer thinking for the yoga teachers and therapists, which is essential to enable them to be on the safer side by avoiding the contraindicated practices. ${ }^{13}$

Yoga teachers, therapists, and practitioners should be motivated to try whether the research studies are "true or not" and are the findings relevant and useful by applying the methodology in their own personal lives. With an increased number of blinded, randomized, and controlled trials, there is ample evidence of yoga research becoming a global phenomenon.
A better understanding of the mechanisms by which various practices cause their effects along with improved planning and implementation is the need of the hour. Standard of research at least at the physical level has been established with an increasing number of research studies getting published in peer-reviewed indexed journals. Greater funding is also available nowadays. ${ }^{7}$

Moreover, the assessment tools, various physiological and biochemical markers, to gauge the health (EEG and evoked potentials for brain activity; heart rate variability measures, blood pressure, ECG and cardiac functions for heart and blood vessels; renal, liver, gastrointestinal, musculoskeletal functions for these specific systems) of various systems of the body might not be sensitive enough to measure the change in these parameters following few weeks of yoga practice.

As the saying goes, "yatho manah tatha prana," yoga as a mindbody harmonizing science improves the "pranic flow of energy." Due to this strong aspect, it becomes justifiable to have a positive and optimistic hypothesis in most of the research work done with yoga.

\section{Weakness of Yoga Research}

As we understand, yoga is not merely performing postures and breath control but it is comprehensive lifestyle changes, and thus, its application and effects are difficult to "prove" what exactly is happening through a research study. Yoga therapists and researchers should be familiar with ongoing research in the fields of yoga, also consider the results with a pinch of salt because of limitations and bias in yoga research.

Diminishing scientific vigor is the major drawback of yoga research in India. Many research studies are being performed to "prove" facts that are known to be "true" and nothing new is proved. This coupled with many limitations such as difficulties in conducting randomized double-blind controlled design, mushrooming of many yoga centers with lack of infrastructure, decreasing integrity 
among academicians with peer pressure to publish more, biased interpretation of positive effects of yoga, and other factors have contributed to questionable scientific validation of yoga research. We need to counter all these weaknesses to strengthen the quality of yoga research in India.

There is a lacuna in routine scientific studies which normally is done for a few weeks or a few months of yoga (asana and pranayama) practice. Hence the qualitative improvement achieved by multidimensional and longitudinal changes in one's own life by following or practicing yoga as a way of life is not usually picked up in this short duration (3-6 weeks/months) of training and practice.

$A$ recent survey done by Bhavanani $A B$ ascertained that most respondents (yoga teachers and therapists) accessed the materials on investigations on yoga through online sources such as articles available on the net, news reports, email communications, and exchanges through their contacts. It was surprising to note that only $7 \%$ of respondents were accessing their information through online official health-related search engines such as MEDLINE or PubMed. On the other hand, $10 \%$ of respondents trusted on various books on the subject to gather information. Anyone depending on the internet sources needs to be diligent with the information available as unsubstantiated sources might convey different messages than in the peer-reviewed publications. ${ }^{13}$

An example to prove this point is from a 2015 study by Lee et al. on the effect of body posture while sleeping on the side alters the brain glymphatic transport thus improper clearing of metabolic excretory substances which in turn results in many neurological disorders. Plenty of arguments followed publishing this article and many scientists started mentioning this article and explaining probable advantages of humans sleeping on one particular side. All these commentators not even concerned to look into the original source and study this published article as this study used rodent models and not human subjects. ${ }^{14}$ Hence simple access to more material might not always result in our increased right knowledge!

As far as yoga research goes, a similar study using maybe similar parameters and same types of standard equipment done at different places across the globe, will the result be similar too? Will that be reliable, reproducible, and valid? More often than not, it becomes extremely difficult to analyze how things happen in different settings world wide, which becomes a confounding factor, and scientific evidence is not available for such research work.

Much of the modern research seems to be directed toward attracting scientists to specific technologies and infrastructure than to developing yoga and ultimately to reach common people enabling them to adopt a yogic lifestyle and get rid of their difficulties.

Another bigger challenge in yoga researches is that yoga being a technique of projecting inward and philosophically inquiring within ourselves. Hence, this is very tough if not impossible to study these processes of "self-inquiry" using the present tools of modern scientific research. Most yoga practices are beyond the effects of various bodily postures and breath control. To decipher that "Shavasana" (corpse posture-a supine relaxation position) has mere the observable effect of reducing blood pressure and heart rate is a very superficial conclusion (tip of an iceberg), missing the real effects of this process of ultimate relaxation. ${ }^{7}$

We still have not designed the facilities or types of equipment to research the higher levels of yoga (mental, spiritual, and philosophical), and most research has been on the asana (bodily postures), pranayama (breath control), and to some extent on the dhyana (meditative) techniques such as transcendental meditation. Further, Indian yoga researchers are restricted by funding and infrastructure, and the majority of the scientific research has been investigating the effects of yoga on basic cardiorespiratory, hypothalamic-pituitary-adrenal axis, and immunological and biochemical parameters.

While doing interventional studies, after collection of the predata analysis and a few yoga training sessions, the subjects disappear. More often than not, they will not be there for postdata collection; hence, dropouts are quite common. Follow-up with the participants is next to impossible. Their work schedule, family pressures, financial burden, etc. become a detrimental factor in their progress. Long-term commitment is required in yoga which is a great weakness.

Funding has always been an obstacle in yoga research, especially in India. Also, people, in general, have this fixated notion in mind that yoga has to be for free. But they forget that the researcher in yoga therapy is undeniably investing his time and energy which is often overlooked.

Yoga is erroneously believed to have come out of Hinduism. Hence, there could be a lot of cultural or religious issues that could come up. The researcher has to respect these modalities in such a way that no one's sentiment is hurt.

\section{Opportunities of Yoga Research}

To counter all the weaknesses and limitations in yoga research, longitudinal studies with multiple parameters (to cover both the width and breadth of specialization, multicentric studies collaborating with various specialties) need to be planned. The entire advanced and existing infrastructure in terms of molecular, genetic, imaging, and clinical diagnostic tools need to be employed to find meaningful conclusions. In addition, investigative measures such as Kirlian photography or other means to visualize auras, chakras, and koshas need to be further examined to be part of this study.

As one digs deeper into research in yoga therapy, along with practical exposure, validations, proofs, etc. the researcher can reach higher up in the ladder. They can work with the doctors, specialists, surgeons, etc. and develop an acceptable regime for the patients, including lifestyle modifications, dietary changes, preparation of practice charts, family counseling, and many more. Ultimately, the research will be of great benefit to the population and the society.

Research in yoga therapy is relatively new but has substantial background and backing behind it. It is more scientific and has enormous psychospiritual assistance. Hence, the potential is huge, and lots of avenues are there to be explored.

In these modern times, therapies have become more "evidence based" rather than just theoretical or philosophical, especially in a medical setting. The terms such as "therapy" and "therapist" have become synonym to "treatment" and "doctor", respectively. Hence, it has become a legal obligation and requires "formal" applications of the therapy. Therefore, nowadays, a therapist has to have valid qualifications in the form of "accepted" degree or diploma or advanced certificates, in addition to having basic knowledge of anatomy and physiology to work with the patients. It makes sense and seems logical that the therapist can work with utmost confidence while working with patients.

Many opportunities exist with medical science as the domain with yoga therapy as an adjuvant, which is still unexplored. Nowadays, any value-based alternate skill is being considered as a career option wherein yoga therapy is an emerging sector, 
and many enthusiastic youngsters are taking up yoga therapy as a career and are showing keen interest in research due to their inquisitiveness.

\section{Challenges of Yoga Research}

To defy all these limitations and shortcomings, one has to build a strong interinstitutional team with like-minded people. Compliance of the study participants to carry on the training protocol is a big concern, and this needs to be dealt with periodic checkups or booster sessions at the center to be built in the design, providing videos of the performance sessions, maintain the diary of practice, few motivational feedbacks. Ultimately "yoga" should be the winner in demonstrating its preventive, promotive, and restorative abilities and complement existing treatment modalities. However, these need to be done in an unbiased way employing rigorous quality checks in the implementation of randomized double-blind controlled study design and protocol. This way we can convince both funding agencies and publish the results in high-impact journals. Hopefully, the yoga research community takes up these challenges positively to explore further the beneficial effects of yoga, its mechanisms thereby helping the mankind.

One of the problems in the current era is that many brilliant scientists are investigating yoga, but because their understanding of this grand science is so limited, they end up missing the bigger picture most of the time. Although nice papers with good scientific backup are published, these are a bit limited from an outlook of yoga.

Many research studies are being published in recent times with mediocre methodologies, wherein lack of safety concerns while performing complicated asanas have been compromised. There has also been over importance given to quantitative issues of research parameters and protocols rather than qualitative aspects of research. Ideally, we need to address more scientific studies combining both aspects of quantity and quality to obtain the optimal output.

Further, there is a lack of scientific awareness regarding yoga research among yoga teachers and therapists. Although a majority of yoga practitioners agree that scientific research in yoga is important, few are attempting to update themselves on such research findings through proper scientifically approved routes (journals and scientific online resources). It is heartening to note that most of the practitioners are updating themselves through general articles on the internet and such vague impacted and not scientifically sound materials seem to have no or negative guidance on their yoga teaching and practice without any significant stimulus to their students or patients in majority of scenarios.

Research in yoga therapy could become expensive when the study requires a lot of tests to be performed, and when there is no funding, it becomes even more difficult. This creates a financial burden on the researcher leading to manipulation of data. Hence this needs to be dealt seriously.

Sometimes feasibility issues in technical, economic, legal, operational, and scheduling keep the researcher on their toes. Apart from these, issues related to logistics, environment, laws and regulations, etc. are also present posing challenges.

It is ideal that yoga teachers follow qualitatively done yoga research studies to update their knowledge as well as to understand the newly arising applications and trends. Most of the yoga teachers and therapists have little idea as how to read a scientific research study or how to differentiate valid information from the sensational but fake news. Hence, most of the time, they tend to infer incorrect and falsified meanings from reading only the abstract or not comprehending the ultimate message. In order for these yoga teachers and therapists to acquire real conclusions from reading yoga research, they need to undergo regular exercise as how to read and comprehend the scientific research articles.

\section{Conclusion}

Students of yoga interested in pursuing with scientific research must qualify themselves in modern science in order to enhance their knowledge in that field which would enable them to interpret the aspects and dimensions of the grand science of yoga in a better manner acceptable by hardcore scientists. In the same line, scientists who investigate yoga, need to understand that yoga is not just an entity to be only "studied" but is also a way of lifestyle to be "lived" and followed every moment. The interpretations need to bring out and highlight the objectivity of the subjective yogic phenomena. An amalgamation of these two grand valuable sciences needs to happen to promote the union of scientific and yogic perspectives so that a wholistic view is achieved.

Overall, the strengths of yoga overpower the weakness, and the opportunities are much stronger than the threats and or challenges.

\section{ACKNOWLEDgments}

The authors thank the management and administrators of SBV for setting up CYTER thus enabling yoga to reach all sections of society in a scientific and holistic manner through the conferences that are organized such as the International Health Research Convention 2019 as a result of which we have been able to compile this paper. We offer our gratitude to all the participants of the seminar cum workshop for their valuable inputs during the open house discussion. Sincere gratitude is also offered to the research scholars and students of CYTER for their wholehearted and generous inputs for this compilation.

\section{References}

1. SWOT Analysis of yoga. Free SWOT analysis. 2019. Available from: https://www.freeswotanalysis.com/health-pharmaceuticalswot/797-swot-analysis-of-yoga.html.

2. Ahmadi Q, Danesh H, Makharashvili V, Mishkin K, Mupfukura L, Teed H, Huff-Rousselle M. SWOT analysis of program design and implementation: a case study on the reduction of maternal mortality in Afghanistan. Int J Health Plann Manage 2016;31(3):247-259. DOI: 10.1002/hpm.2288.

3. Miller MG. Environmental metabolomics: a SWOT analysis (strengths, weaknesses, opportunities, and threats). J Proteome Res 2007;6(2):540-545. DOI: 10.1021/pr060623x.

4. Teoli D, An J. SWOT analysis. 2019. StatPearls [Internet]. Treasure Island (FL): StatPearls Publishing; 2019. Available from: http://www-ncbinlm-nih. PubMed PMID: 30725987.

5. Von Kodolitsch Y, Bernhardt AM, Robinson PN, KölbelT, Reichenspurner $\mathrm{H}$, Debus S, Detter C. Analysis of strengths, weaknesses, opportunities, and threats as a tool for translating evidence into individualized medical strategies (I-SWOT). Aorta (Stamford) 2015;3(3):98-107. DOI: 10.12945/j.aorta.2015.14.064.

6. Wendling, DL, editor. Bethesda (MD): National Library of Medicine (US); 2007. Available from: http://www.nlm.nih.gov/ citingmedicine. 
7. Bhavanani $A B$. Yoga research - what are we doing? In: Bhavanani $A B$, editor. Notes on anatomy \& physiology for yoga education. Pondicherry, India: CYTER of Sri Balaji Vidyapeeth; 2018. p. 172-178.

8. Bhavanani $A B$. Yoga Chikitsa: the application of yoga as a therapy. Pondicherry, India: Dhivyananda Creations; 2013.

9. Jeter PE, Slutsky J, Singh N, Khalsa SB. Yoga as a therapeutic intervention: a bibliometric analysis of published research studies from 1967 to 2013. J Altern Complement Med 2015;21(10):586-592. DOI: 10.1089/acm.2015.0057.

10. Tang H, Huang W, Ma J, Liu L. SWOT analysis and revelation in traditional Chinese medicine internationalization. Chin Med 2018;13:5. DOI: 10.1186/s13020-018-0165-1.
11. SWOT analysis [Internet]. En.wikipedia.org. 2019. Available from: https://en.wikipedia.org/wiki/SWOT_analysis.

12. Feuerstein G. Yoga therapy: a complete system of preventive, curative and promotive health. In: Bhavanani $A B$, editor. Principles and methods of yoga therapy: study materials. Pondicherry, India: Dhivyananda Creations; 2010. p. 1-4.

13. Bhavanani $A B$. A brief qualitative survey on the utilization of yoga research resources by yoga teachers. J Intercult Ethnopharmacol 2016;5(2):168-173. DOI: 10.5455/jice.20160331064758.

14. Lee $H$, Xie L, Yu M, Kang H, Feng T, Deane R, et al. The effect of body posture on brain glymphatic transport. J Neurosci 2015;35(31):1103411044. DOI: 10.1523/JNEUROSCI.1625-15.2015. 